\title{
Digital Riemannian Geometry and Its Application
}

\author{
Guohua Chen \\ School of Med-Info Engineering, Guangdong Pharmaceutical University, Guangzhou 510224, P.R.China \\ ghchen2007@yahoo.com.cn
}

\begin{abstract}
The key characteristic of Riemannian geometry is Riemannian metric. The most important work for the discretion of Riemannian geometry is to seek a discret representation of Riemannian metric, which forms the foundation of digital Riemannian geometry. This paper initiated the conception of Digital Riemannian geometry which imposes a Riemannian metric on a rectangle grid to make them curved and induced a weighted distance on them. The main task of Digital Riemannian geometry is to obtain quantitative information about objects in pictures with the help of a discrete Riemannian metric defined on them.
\end{abstract}

Keywords-Digital Riemannian geometry, Digital Finsler geometry, Digital Sobolev geometry, Image processing, Active contour models, Edge detection.

\section{INTRODUCTION}

Digital geometry is now a well known mathematical discipline studying the geometric properties of digital objects (usually modeled by sets of points with integer coordinates) and providing methods for solving various problems defined on such objects.

The history of digital geometry can be traced back to 70 's last century. Although digital geometry was rooted in several classical disciplines (such as topology, graph theory, number theory, and Euclidean and analytic geometry), it was established as an independent subject only in the last few years. According to the definition given in the book "Digital Geometry"[1] by Azriel Rosenfeld and Reinhard Klette: "Digital geometry is the study of geometric or topologic properties of sets of pixels or voxels. It often attempts to obtain quantitative information about objects by analyzing digitized (2D or 3D) pictures in which the objects are represented by such sets". This definition emphasizes especially on the analysis of digital images. Digital geometry had been developed with the explicit goal of providing rigorous mathematical foundations and basic algorithms for applied disciplines such as computer graphics, medical imaging, pattern recognition, image analysis and processing, image understanding, computer vision, and biometrics. Digital geometry focuses mainly on digital pictures, especially on 2D pictures. Taking picture is a process of projecting or flattenning a surface onto a $2 \mathrm{D}$ plane. Each pixel on the image plane has only two coordinates $(\mathrm{x}, \mathrm{y})$ to represent a correspondent 3D point on the surface. Though the reverse process of flattening is an ill-posed problem, the projected pixel contains rich information about original 3D point on the surface, which survived in the process of flattening due to the lightening modes and hidden in the intensity of the pixel. Digital Riemannian geometry (DRG) can curvalize a picture to expose its original geometric information, a partial reverse process of flattening, which is the key characteristic of DRG.

Image curvaliztion is in nature equivalent to assigning a metric on the image plane. An image is generally defined on a rectangle grid. There are already many metrics on a grid space. But those traditional metrics are image independent and so are not proper for image analysis. We need image-related metrics for the purpose of image analysis.

The key problem of DRG is to define a reasonable discrete Riemannian metric on a grid and calculate it.

\section{TRADITIONAL METRICS ON A GRID}

\section{A. Base Space of DRG and traditional metric on a regular $2 D$ grid}

The base space of DRG is in general a rectangle grid $G$ on 2D plane or 3D space such as showed in Fig.1. We will use the representation (a) of Fig. 1 in the following text. The base space forms a grid graph on the plane. Each coordinate point $(x, y)$ in grid $G$ is called a pixel or voxel. The sampled image $\mathrm{I}(\mathrm{x}, \mathrm{y})$ forms a $\mathrm{M} \times \mathrm{N}$ matrix.

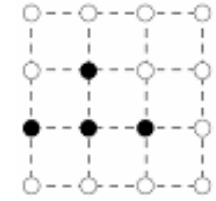

(a)

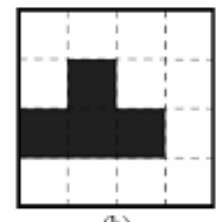

(b)

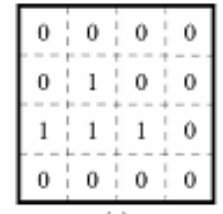

(c)
Fig.1.Grid $\mathrm{G}$ and Representation of grid elements

Traditional metrics on a $2 \mathrm{D}$ grid $\mathrm{G}$ include city-block distance, chessboard distance and Euclidian distance. They are defined as follows:

If $\mathrm{u}, \mathrm{v}$ are two node in the grid $\mathrm{G}$ with coordinate $(\mathrm{x}, \mathrm{y})$, $(\mathrm{s}, \mathrm{t})$ respectively, then

(1) The Euclidean distance of $u, v$ is defined as:

$$
D_{e}=\left[(x-s)^{2}+(y-t)^{2}\right]^{1 / 2}
$$

(2) The city-block distance of $u, v$ is defined as:

$$
D_{4}(p, q)=|x-s|+|y-t|
$$

(3) The chessboard distance of $u, v$ is defined as:

$$
D_{8}(p, q)=\max (|x-s|,|y-t|)
$$

These metrics are image independent and so are not good enough for image analysis. We need an image-related metric which assigns weights on the edges of the grid graph. The weighted metric can be considered as a kind of discrete Riemannian metric. But before a Riemannian metric could be defined on a regular grid graph $\mathrm{G}$, the neighborhood 
system on grid graph must be revised so that we can define a weighted distance between two nodes in the grid.

\section{B. Neighborhood systems on a regular $2 \mathrm{D}$ grid}

In this section we will follow Boycov's terminologies [2-3]. We restrict our discussion on $R^{2}$ only. Assuming the grid graph with cells of size $\delta$ and that all nodes have identical neighborhood systems topologically. The figure 2 bellow shows some examples of possible neighborhood systems.

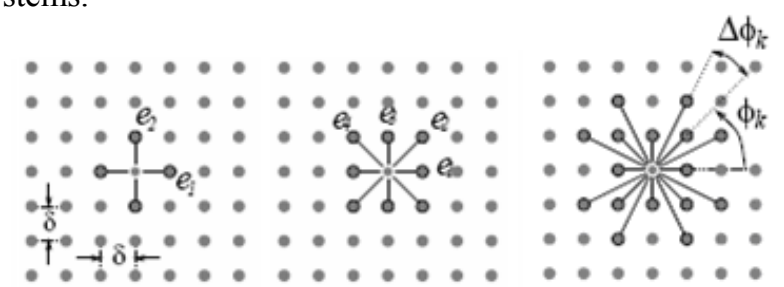

(a)

(b)

(c)

Fig.2. Examples of neighborhoods in 2D.

(a) 4n-system (b) 8n-system (c) 16n-system

A neighborhood system on a regular grid can be described with a set: $N_{G}=\left\{e_{k}: 1 \leq k \leq n_{G}\right\}$, where $e_{k}$ 's are distinct undirected vectors.

For example, grids with a $8 \mathrm{n}$ neighborhood system can be described by the set of four vectors $N_{G}=\{\mathrm{e} 1, \mathrm{e} 2, \mathrm{e} 3, \mathrm{e} 4\}$ as shown in Figure 2(b). We assume that vectors $e_{k}$ 's will be enumerated in an increasing order of their angular orientation $\varphi_{k}$ so that $0=\varphi_{1}<\varphi_{2}<\cdots<\varphi_{n_{G}}<\pi$. For ease of discussion, we will assume in the following that $e_{k}$ is the shortest length vector connecting two grid nodes in the given direction $\varphi_{k}$. Each vector $e_{k} \in N_{G}$ generates a family of edge-lines on the corresponding grid. The distance between the nearest lines in a family generated by $e_{k}$ is:

$$
\Delta \rho_{k}=\frac{\delta^{2}}{\left|e_{k}\right|}
$$

where $\delta$ is the cell-size of the grid and $\left|e_{\kappa}\right|$ is the (Euclidean) length of vector $e_{\kappa}$. Each family of edge lines is characterzed by the inter-line distance $\Delta \rho_{k}$ and by its angular orientation $\varphi_{k}$. The angular differences, $\Delta \varphi_{i}=\varphi_{i+1}-\varphi_{i}, i=1, \ldots, N_{G}$, between the nearest families of edge lines will be used later.

The weights on its edges is another important aspect of a grid graph. All edges in the same family of lines will be set the equal weights. This means that, for all edges with orientation $\varphi_{k}$, we will use $w_{\kappa}$ to denote their common weights. Suppose $\mathrm{G}=<\mathrm{V}, \mathrm{E}>$ is a weighted graph. For any path $P_{S T} \subset E$ connecting two nodes $\mathrm{S}, \mathrm{T} \in \mathrm{V}$ in $\mathrm{G}$, Length of the path can be defined naturally as the sum of edge weights along the path:

$$
\left|P_{S T}\right|=\sum_{e \in P_{S T}} w_{e}
$$

A (s-t)-cut on a grid-graph $\mathrm{G}=<\mathrm{V}, \mathrm{E}>$ embedded in $R^{n}$ can be seen as a closed contours (in $R^{2}$ ) or as a closed surfaces (in $R^{n}$ ). For any s-t cut $C \subset E$, its (cut) length, or area in $R^{n}$, can be defined naturally as:

$$
|C|_{G}=\sum_{e \in C} w_{e}
$$

This is simply the standard definition of cut cost from combinatorial optimization. Due to geometric interpretation of $|C|_{G}$ as the "length" or "area" of the corresponding contour or surface, we call it the cut metric of contour C.

A cut metric on a regular $2 \mathrm{D}$ grid implicitly assigns certain "length" to curves. If we use $|C|_{E}$ to denote the Euclidean length of the curve $C$ in $G$, then we can use Cauchy-Crofton formula[4] to approximate it by:

$$
|C|_{E} \approx \frac{1}{2} \sum_{k=1}^{n_{G}}\left(\sum_{i} n_{c}(i, k) \Delta \rho_{k}\right) \Delta \Phi_{k}=\sum_{k=1}^{n_{G}} n_{c}(k) \frac{\delta^{2} \Delta \Phi_{k}}{2\left|e_{k}\right|}
$$

here index $\mathrm{i}$ enumerates all the lines in the kth family of edge-lines. $n_{C}(i, k)$ counts intersections of line $i$ in the kth family with contour $C . \mathrm{n}_{c}(k)=\sum_{i} n_{c}(i, k)$ is the total number of intersections of $C$ with the kth family of edge-lines. If we choose constant edge weights within each family of edge lines as:

$$
w_{k}=\frac{\delta^{2} \Delta \Phi_{k}}{2\left|e_{k}\right|}
$$

then we have:

$$
|C|_{E} \approx \sum_{k=1}^{n_{G}} n_{C}(k) \cdot w_{k} \approx|C|_{G}
$$

If the 2D space is assigned a Riemannian metric, then an induced weights will be assigned to grids imbedded in it, and a contour curve $\mathrm{C}$ in it will have its Riemannian length $|C|_{R}$. If we assigning proper weights to the edges of the grid graph, the cut metric of the contour $\mathrm{C}$ will approximate its Riemannnian metric, i.e. $|C|_{R} \approx|C|_{G}$. In fact, if the Riemannian 2D space has a constant metric $\mathrm{D}\left({ }^{*}\right)=$ const. i.e. 
$\mathrm{D}$ is a constant matrix. For a contour $\mathrm{C}$ with Riemannian length $|C|_{R}$, there is a Crofton-typed formula as following:

$$
|C|_{R}=\frac{1}{2} \int \frac{\operatorname{det} D}{2\left(u_{L}^{T} \cdot D \cdot u_{L}\right)} n_{C} d L
$$

here $u_{L}$ is a unit vector along line L. This formula holds for any continuously differentiable regular curve $\mathrm{C}$ in $\mathrm{R}^{2}$. If we set edge weights for a 2D grid as follows:

$$
w_{k}=\frac{\delta^{2}\left|e_{k}\right|^{2} \Delta \Phi_{k} \operatorname{det} D}{2\left(e_{k}^{T} \cdot D \cdot e_{k}\right)^{\frac{3}{2}}}
$$

Then we have $|C|_{R} \approx|C|_{G}$.

A general Riemannian metric is a tensor $\mathrm{D}(\mathrm{p})=\left(g_{i j}\right)[5]$. If $\mathrm{G}$ is looked as a directed graph and the weights are assigned to its edges, then the cut metric on $G$ is called a digital or discrete Riemannian metric on G. Here we use $w_{k}(p)$ to denote the weight of a directed edge originated from p. Digital Riemannian geometry attempts to obtain quantitative information about objects in pictures with the help of a digital Riemannian metric defined on them.

$$
w_{k}(p)=\frac{\delta^{2}\left|e_{k}\right|^{2} \Delta \Phi_{k} \operatorname{det} D(p)}{2\left(e_{k}^{T} \cdot D(p) \cdot e_{k}\right)^{\frac{3}{2}}}
$$

\section{IMAGE CURVALIZATION AND INDUCED METRICS}

Image segmentation is a process of partitioning image plane into meaningful areas which are connected and without overlaps between each other $[6,15]$. Meaningful mainly refers to a separation of areas corresponding to different objects in the observed scene from the area corresponding to the background. Image segmentation is very fundamental in image processing. Many other techniques, such as characteristics extraction, auto-detection or objective tracing, are based on the results of segmentation. Image segmenting techniques can be classified into many categories according to different standards. Typical classification includes edge detection and area splitting and emerging. Traditional method of edge detection is through thresholding values. This method lacks a well defined mathematical model. It treats the boundary of an object not as a whole but as a scattered set of points with intensities greater or less than the threshholding value. A noticeable edge detection method with good mathematical model is the Active Contour Models introduced by Kass[14]. It is also known as "Snake Model".
An active contour is a curve $C(t)=(x(t), y(t)), t \in[0,1]$, which moves in an image to make the energy $E(C)$ minimized:

$$
E(C)=K_{0} \int_{0}^{1}\left\|C^{\prime}(t)\right\|^{2} d t+K_{1} \int_{0}^{1}\left\|C^{\prime \prime}(t)\right\|^{2} d t+\int_{0}^{1} P(x(t), y(t)) d t
$$

here $\mathrm{P}(\mathrm{x}, \mathrm{y})$ is a potential function, $K_{0}$ and $K_{1}$ are tension and rigidity parameter of the curve respectively. Calculus of variation $[8,17]$ had proved that the curve $C$ that minimize $E(C)$ should satisfy the Euler equation[14]:

$$
-\nabla P(C(t))+2 K_{0} C^{\prime \prime}(t)-2 K_{1} C^{\prime \prime \prime}(t)=0
$$

In which we suppose that $C(\cdot)$ and its derivatives are known for $\mathrm{t}=0$ and $\mathrm{t}=1$.

A main weakness of Kass's model is that the energy expression (10) depends on parametrization. Many improvements had been proposed to elimilate the dependence of the energy on parametrization [8-12]. The one proposed by Caselles et.al. [9] attracted many followers, which is called "Geodesic Active Contour Model" and dose not depend on the parametrization. In Caselles' model, the rigidity parameter was taken as 0 , i.e. $K_{1}=0$, and $\mathrm{P}(\mathrm{x}, \mathrm{y})=h(|\nabla I(x, y)|)^{2}$, so the equation (10) becomes as :

$$
E(C)=\alpha \int_{0}^{1}|C(t)|^{2} d t+\lambda \int_{0}^{1} h(|\nabla I(C(t))|)^{2} d t
$$

here $h:[0,+\infty] \rightarrow R^{+}$is a strictly decreasing function, such that $h(\mathrm{r}) \rightarrow 0$ when $\mathrm{r} \rightarrow \infty$. If a proper $\alpha, \lambda$ is taken, we can transform the minimization probrem of $E(C)$ to the intrinsic minimal problem of following form:

$$
\operatorname{Min}\left\{E(C)=\int_{0}^{1} h(|\nabla I(C(t))|)\left|C^{\prime}(t)\right| d t\right\}
$$

If we introduce a Riemannian metric $\mathrm{g}$ on image plane I as $g_{i j}=h(|\nabla I(x)|)^{2} \delta_{i j}$. Then to solve equation (13) is equivalent to seek for the minima of $E(C)$ in following formula:

$$
E(C)=\int_{0}^{1} \sqrt{g_{i j} \dot{x}^{i} \dot{x}^{j}} d t \doteq|C|_{R}(\mathrm{i}, \mathrm{j}=1,2)
$$

here $C_{t}=\left(\dot{x}^{1}(t), \dot{x}^{2}(t)\right)$. So, after the introduction of the Riemannian metric $\mathrm{g}=\left(g_{i j}\right)$ on the image plane I, to detect an edge in $\mathrm{I}$ is equivalent to seek a curve in the Riemannian space I with minimal weighted length $|C|_{R}$. The image plane I can now be thought as curvalized. 
Formally speaking of curvalization, a grayscale image $I(x, y)$ is considered as a surface $M$ in $R^{3}$, with its domain contained in the parametric plane $U(x, y)$. If we set $\mathrm{x} 1=\mathrm{x}, \mathrm{x} 2=\mathrm{y}$, then the parametric plane $U\left(x^{1}, x^{2}\right)$ is mapped into a surface $M$ in $R^{3}$ via X:

$$
\begin{aligned}
X & : U \rightarrow M \subset R^{3} \\
\left(x^{1}, x^{2}\right) & \mapsto\left(x^{1}, x^{2}, I\left(x^{1}, x^{2}\right)\right) \in R^{3}
\end{aligned}
$$

$M$ and $U$ can both be considered as Riemannian manifolds [See Fig.3]. $M$ is the image space, $U$ is the domain space. Any curve $C(t) \in X(U)$ is mapped from a curve $\tilde{C}(\tilde{t}) \in U$, i.e. each point $\mathrm{p} \in C(t)$ is a mapping of a corresponding point $\tilde{p} \in \tilde{C}(\tilde{t}), \mathrm{p}=X(\tilde{p})$.

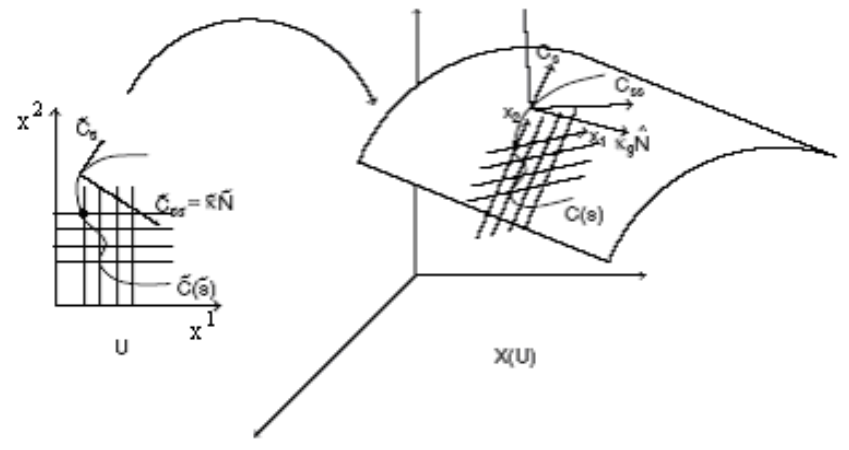

Fig.3. A curve $C(t)$ on the surface $M$ and its origin $\tilde{C}(\tilde{t})$ on the parameterization plane $U$

The typical metric on $U$ is the ordinary euclidean metric $d s^{2}=\left(d x^{1}\right)^{2}+\left(d x^{2}\right)^{2}$. The typical metric on $M$ is induced from $R^{3}$ as follows:

$$
\begin{gathered}
d s^{2}=\left(d x^{1}\right)^{2}+\left(d x^{2}\right)^{2}+d I^{2} \\
=\left(1+I_{1}^{2}\right)\left(d x^{1}\right)^{2}+2 I_{1} \cdot I_{2} d x^{1} d x^{2}+\left(1+I_{2}^{2}\right)\left(d x^{2}\right)^{2}
\end{gathered}
$$

here $I_{i}=\frac{\partial I}{\partial x^{i}}$. Let $\frac{\partial}{\partial x^{1}}=\left(1,0, \frac{\partial I}{\partial x^{1}}\right), \frac{\partial}{\partial x^{2}}=\left(0,1, \frac{\partial I}{\partial x^{2}}\right)$, then $\frac{\partial}{\partial x^{1}}, \frac{\partial}{\partial x^{2}}$ form a basis on the tangent plane of $M$. The induced metric on $M$ from $R^{3}$ can be denoted as $d s=\sqrt{g_{i j} d x^{i} d x^{j}}$, the Einstein summation convention was used here. The covariant variables $g_{i j}$ on $M$ are defined as follows:

$$
\begin{aligned}
& g_{11}=<\frac{\partial}{\partial \mathrm{x}^{1}}, \frac{\partial}{\partial \mathrm{x}^{1}}>_{d s^{2}}=1+I_{1}^{2}, g_{22}=<\frac{\partial}{\partial \mathrm{x}^{2}}, \frac{\partial}{\partial \mathrm{x}^{2}}>_{d s^{2}}=1+I_{2}^{2} \\
& g_{12}=g_{21}=<\frac{\partial}{\partial \mathrm{x}^{1}}, \frac{\partial}{\partial \mathrm{x}^{2}}>_{d s^{2}}=I_{1} \cdot I_{2}
\end{aligned}
$$

For the map $\mathrm{X}$ above, it can produce a pulled back metric on $U$ via pull back mapping $X^{*}[13]$.
Define a proper Riemannian metric $\mathrm{g}$ on an image plane I is not intuitive and even very difficult. We can instead define a metric on the curved space $M$ and then pull it back to I, which can often highlight characteristics of an image. For instance, an anistropic Riemannian metric induced by an image I can be represented as:

$$
D(p)=\lambda(|\nabla I|) I d+(1-\lambda(|\nabla I|)) \vec{u} \cdot \vec{u}^{T}
$$

here $\vec{u}=\nabla I /|\nabla I|$ is a unit vector along the gradiant at a pixel of I. Id is the identity matrics, $\lambda(|\nabla I|)$ is a scalar function. In the coordinate system align with gradiant (then $\left.\vec{u}^{T}=(1,0,0)\right)$, metric $D(p)$ can be represented as a diagonal matrix: $D(p)=\operatorname{diag}(1, \lambda(|\nabla I|), \lambda(|\nabla I|))$.

For the purpose of image analyse, we need such metrics to be defined on the base space of an image that it can enlarge some particular characteristics of the image, such as edges or points with rapid gradiant variations. With the help of the idea of image curvalization, many powerful tools from Riemannian geometry had found their applications in image processing. Motivated by certain problems in pattern detection[18] and medical imaging, Melonakos et.al.[16] developed a kind of active contours model with a Finsler metric. Its main idea is that the directionality be added to the active contours to allow for the segmentation of images from oriented domains. Sundaramoorthi et.al. introduced the concept of Sobolev Active Contour Model, which re-formulate the general geometric active contour by re-defining the gradient of functional with a Soloblev-typed inner product. More details are refered to the papers [19-21].

\section{CONCLUSION}

Digital geometry had developed for quite a long period and played a more and more important role in image processing. Recent years, many researchers had tried to solve various problems with the help of Riemannian geometry. Part of these endeavors had achieved success. In this paper, we briefly introduced some basic ideas of DRG. It should be noticed that not only finite dimensional Riemannian manifolds had been digitized, digital development of infinite dimentional Hilbert manifolds had also attracted many attentions and developed even more rapidly [7]. DRG had emerged as a new subject and showed great promise in diverse applications of image science.

\section{ACKNOWLEGEMENT}

This work was done under the support of the Natural Science Foundation of Guangdong Province, China (Grant No. S2011010001841).

\section{REFERENCES}

[1] R. Klette and A. Rosenfeld: Digital Geometry: Geometric methods for digital picture analysis. Elsevier (Singapore) Pte Ltd. Singapore, 2006. 
[2] V.Boycov, O. Veksler, R.Zabih: Fast Approximate Energy Minimization via Graph Cuts. IEEE Trans. PAMI, Vol.23, No.11, 2001

[3] Y. Boykov, V. Kolmogorov: Computing Geodesics and Minimal Surfaces via Graph Cut. In Proc. IEEE Int. Conf. on Computer Vision. 2003, 26-33

[4] B. Su: Five lectures in differential Geometry. Shanghai Sci. Pub. Co., 1979

[5] S. Lang: Fundamentals of Differential Geometry. Springer-Verlag. 1999

[6] D. B.Karron, J. Cox.: Extracting 3D objects from volume data using digital morse theory, Computer Vision, Virtual Reality and Robotics in Medicine (N. Ayache, ed.), No. 905 of Lecture Notes in Computer Science, (New York), 1995, 481-486, Springer-Verlag..

[7] P. W. Michor, D. Mumford: An overview of the Riemannian metrics on spaces of curves using the Hamiltonian approach. Appl. Comput. Harmon. Anal. 23 (2007) 74-113

[8] R. Goldenberg, R. Kimmel, E. Rivlin, et al: Cortex Segmentation: A Fast Variation Geometric Approach, IEEE Transactions on Medical Imaging, 2002, 21(2): 1544-1551.

[9] V. Caselles, R. Kimmel, G. Sapiro: Geodesic Active Contour. International Journal Of Computer Vision 1997, 22(1), 61 79

[10] L.D. Cohen: On active contour models and ballons. Comput.Vision, Graphics, and Image Processing: Image Processing, 1991, 53(2).

[11] N. Paragios, R. Deriche: Geodesic active contours and level sets for the detection and tracking of moving objects.IEEE Trans.On Patt.Anal.and Mach. Intell.,2000,22(3):266-280

[12] N. Paragios, R. Deriche: Geodesic active regions: A new paradigm to deal with frame partition problems in computer vision. International
Journal of Visual Communication and Image Representation, Special Issue on Partial Differential Equations in Image Processing, Computer Vision and Computer Graphics, 2002, 13(2):249-268.

[13] X. Gu, Y. Wang , S.-T. Yau: Computing Conformal Invariants: Period Matrics. Communication in Information and System, Vol.3, No.3, 2004, 153-170.

[14] M. Kass, A. Witkin, D.Terzopoulos: Snakes:Active contour models. In Proc. IEEE Int. Conf. on Computer Vision 1987, 259-268.

[15] D. Mumford, J. Shah.: Optimal approximations by piecewise smooth functions and associated variational problems. Comm. Pure Appl. Math., 1989, 42:577-685.

[16] V. Mohan, J. Melonakos, A. Tannenbaum, M. Niethammer, M. Kubicki: Finsler Level Set Segmentation for Imagery in Oriented Domains. BMVC2007,1-9.

[17] O. Faugoras, R. Keriven: Variational principles, surface evolution, PDE's Level Set methods, and the stereo problem, IEEE Transactions on image Processing, 1998,7(3):336-344.

[18] R.O. Duda, P. E. Hart, D. G. Stork: Pattern classification (2nd edition), Wiley, New York. 2001.

[19] A. Mennucci, A. Yezzi, G. Sundaramoorthi: Sobolev-type metrics in the space of curves. arXiv:math/0605017v1 [math.DG] 30 Apr 2006

[20] Ye Yuan, Chuanjiang He : Variational level set methods for image segmentation based on both $\mathrm{L}^{2}$ and Sobolev gradients. Nonlinear Analysis: Real World Applications 13 (2012) 959-966

[21] Sundaramoorthi G.., Yezzi A., Mennuci A., Sapiro G.: New Possibilities With Sobolev Active Contours, Int J Comput Vis (2009) 84: 113-129 